\title{
What Does It Take to Get Published?
}

\author{
C. V. Hegde ${ }^{1}$
}

Received: 6 February 2017 / Accepted: 6 February 2017/Published online: 6 March 2017

(C) Federation of Obstetric \& Gynecological Societies of India 2017

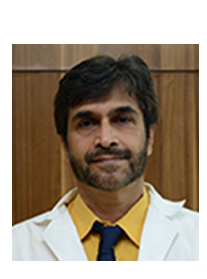

\begin{abstract}
About the Author
Dr. C. V. Hegde is Editor in Chief of "The Journal of Obstetrics and Gynecology of India". He is also Professor and Chief of Unit at the T N Medical College and BYL Nair Hospital, Mumbai. He is also Visiting Consultant at the Sir HN Reliance Foundation Hospital, Mumbai. He is an Endoscopic surgeon at the St. Elizabeth's Hospital, Mumbai. He has had a rich teaching experience of more than three decades and has taught and trained a large number of undergraduate and postgraduate students in that duration of time. He has been a postgraduate and undergraduate examiner in Obstetrics and Gynecology at the Bombay University and the Maharashtra University of Health Sciences. He runs the PICSEP program of the Journal and FOGSI under whose aegis ten programs in research methodology are conducted all over the country every year. This program aims at improving the quality of original research papers being submitted to the journal. He has several research papers published in this journal and several other journals of standing. He is the winner of the prestigious FOGSI Corion award in the senior category. He has more than 50 videos on the YouTube on all aspects of vaginal and endoscopic surgery, solely for the purpose of furthering
\end{abstract} medical education. His other interests include non-fiction reading and reading blogs on Indian history and contemporary politics.

The first question that arises is-why? I suppose the most important reason would be that the researcher has reached some exciting conclusions after an arduous study that he/ she feels would make a difference. Mundane reasons would include "publish or perish" as the guiding force, especially if advances in career depend upon the publication of original research articles in reputed indexed journals at regular intervals. A desire to be well known in the research world could well be another reason. Whatever be the reason, in order to generate hypotheses to start the study, a driving force is required in the form of regular reading of contemporary articles from different sources, attending

\footnotetext{
C. V. Hegde

cvvh29@yahoo.co.in
}

1 Mumbai, India seminars, conferences or an in-depth study of an application of a nuanced treatment to a commonly occurring ailment, etc. The seed of an idea to generate hypotheses is then planted.

Nurturing this idea with inputs from other independent researchers (who could give a negative feedback as well) is the next step. The hypotheses generated should be topical, contemporary and interesting enough to have the impetus to give it shape in the form of collecting data after a proper study design has been arrived upon.

Retrospective observational studies like case reports or case series report are at the bottom end of the scale in research methodology. Most reputed indexed journals seldom publish case reports. Other observational analytical studies include case-control studies wherein the outcome is already known (e.g. cataract) and where cases and similar controls are studied by going back in time to ascertain risk 
factors (e.g. diabetes). Titles of case-control studies would be as- "Risk factors in preterm delivery/Risk factors in deep vein thrombosis and pulmonary embolism. A population based case control study". Cross-sectional studies try to define "prevalence", the detection of a number of cases at a point in time. Most articles submitted for publication are cross-sectional observational studies which are rather low down in hierarchal evidence-based studies. Examples include "Prevalence of HIV in pregnant women registered at a tertiary hospital" or "A hospital based study on the prevalence of gestational diabetes mellitus".

Cohort studies too are observational studies and include cases with potential risk factors which are matched with similar controls and followed up over a period of time to note an outcome. Cohort or longitudinal studies are often prolonged with a high attrition or falling out rate. Too much attrition leads to data getting compromised and the study getting abandoned. Examples of cohort studies are "Obesity and the risk of stillbirth: a population based cohort study", "Moderate caffeine consumption and the incidence of preterm birth". Cohort studies are valuable in the sense that "incidence" or detection of new cases due to existing risk factors is obtained. Interventional studies include randomised control trials (RCTs) and clinical trials. RCTs provide the highest level of evidence.

The next step involves the collection of data which could be nominal, ordinal, interval and ratio. The statistician should be involved at this phase of research. After studying the type of data to be collected, the statistician would then decide upon the sample size including the method of data collection, the statistical tests to be employed and the software to be used. Often the statistician is involved at the very end of the study when a salvage job is attempted in desperation.

Instructions to authors vary with each journal and must be rigidly adhered to. Upon submission, the article is usually put through the wringer in the Editorial Manager to rule out plagiarism in any form. Plagiarism is a serious offence and may lead to consequences to the offending party in myriad ways besides naming and shaming alone. The article is then read for its "readability" factor. Per se the article has to be topical and interesting to readers. Articles like retrospective studies in institutions on maternal mortality, rupture uterus, chronicles in obstetric calamities, etc., are often rejected immediately because frailties of management are the only factors that stand out. No action taken reports are submitted which make the exercise aimless. In general, retrospective studies are of no great research value and rejection is to be expected. The features that are looked for immediately include seeing whether there is any purpose for the research in the first place. The title of the article should be a mini-summary of the research material. Any flamboyant title is often looked upon with suspicion. The conclusions need to match the aims. The structured abstract should be precise and concise to meet these requirements. Oftentimes the reader interest falls off by about $40 \%$ and the rest of the article ignored if the title and structured abstract fails to evince interest in the reader. The timeline is then noted, and "dated" articles are summarily rejected. A wrong study design and/or wrong application of statistics results in the article being returned for a complete overhaul. Bibliography should be as per the "Vancouver" method. If the initial scrutiny of the article meets requirements broadly the article is then sent for double-blind two peer reviews. The remarks of the peer reviewers are always considered whilst an editorial decision regarding acceptance/rejection is made; however, their comments are not binding as far as the editors are concerned. After the peer review test, an article considered for publication is gone through with a fine toothcomb and clarifications if any are sought from the author. It is in the interest of the authors to respond as soon as possible to indicate their seriousness of purpose.

Articles where the aims are vague and conclusions not laid out precisely, where study designs are awry, where the methods for sampling is not detailed, where wrong statistics is applied, where conclusions are brazenly drawn and recommendations are made recklessly, where the analysis is completely at odds with the data and where "cause" and "effect" are determined by descriptive observational studies are rejected.

A basic adherence to the principles of research methodology will go a long way in ensuring that an original article submitted for publication sees the light of day. 